\title{
Mechanical Performance of Additively Manufactured Fiber-Reinforced Functionally Graded Lattices
}

\author{
JÁNOS PLOCHER (1) ${ }^{1,2}$ and AJIT PANESAR (i) ${ }^{1}$ \\ 1.-Department of Aeronautics, Imperial College London, London SW7 2AZ, UK. 2.-e-mail: \\ janos.plocher16@imperial.ac.uk
}

Latticing has become a common design practice in additive manufacturing $(\mathrm{AM})$ and represents a key lightweighting strategy to date. Functional graded lattices (FGLs) have recently gained immense traction in the AM community, offering a unique way of tailoring the structural performance. This paper constitutes the first ever investigation on the combination of graded strut- and surface-based lattices with fiber-reinforced AM to further increase the performance-to-weight ratio. The energy absorption behavior of cubic lattice specimens composed of body-centered cubic and Schwarz-P unit cells with different severities of grading but the same mass, considered for uniaxial compression testing and printed by fused deposition modelling of short fiberreinforced nylon, were investigated. The results elucidate that grading affects the energy absorption capability and deformation behavior of these lattice types differently. These findings can provide engineers with valuable insight into the properties of FGLs, aiding targeted rather than expertise-driven utilization of lattices in design for AM.

\section{INTRODUCTION}

Latticing has become a widely adopted design approach to lightweighting ${ }^{1-3}$ in additive manufacturing (AM). Taking advantage of the virtually unconstrained design freedom offered by AM, lattices can provide unique solutions to common engineering problems. Besides improving the buckling load ${ }^{4}$ or strength-to-weight ratio ${ }^{5}$ compared with their solid counterparts, lattices are also well suited for multiphysics problems dealing with heat dissipation $^{6,7}$ or energy absorption. ${ }^{8,9}$ An array of unit cell libraries is at the engineer's and designer's disposal today, ${ }^{10,11}$ offering unique mechanical performance. To generate cellular structures and introduce them into the design process, a number of specialized software packages are available industrially. ${ }^{12-15}$ However, implementation of lattices today remains strongly expertise driven due to the limited database at the engineer's disposal. In this context, it is important to realize that substituting solids with lattices can only be done for parts with an already high safety factor, as solids will always outperform their porous counterparts when it comes to stiffness. Latticing is therefore solely a design approach for lightweighting rather than a stiffnessoptimal approach, like e.g. mathematically driven topology optimization, which has provided more reliable predictions of performance to date. Research into lattices has, therefore, attracted immense interest over recent years to investigate the behavior and performance of various cellular configurations. The results of such study will help to improve predictions of their properties and ultimately build confidence in their application for critical parts in the future.

Generally, lattice unit cells are differentiated into strut and surface based, such as body-centered cubic (BCC) and face-centered cubic or Gyroid and Schwarz-P (SP), respectively. A group of surface-based unit cells-also found widely in nature ${ }^{16}$-are the triply periodic minimal surface (TPMS) lattices, ${ }^{11,17}$ which can be represented (1) implicitly by employing level sets, (2) parametrically or (3) through boundary representations. ${ }^{18}$ Universal to all TPMS is their zero mean curvature and a periodicity function specifying unit cell tessellations in three dimensions. ${ }^{19,20}$ From these unit cells, lattices are commonly created by tessellation, sweeping, or mapping. 


\section{Functionally Graded Lattices (FGLs)}

Functionally graded lattices (FGLs) represent an emerging topic in academia, with research ranging from theoretical modeling ${ }^{19,21,22}$ to experimental testing. ${ }^{23-26}$ By changing the local density, FGLs provide advanced customizability in terms of structural response and performance. From early works, realizing stepwise grading ${ }^{23}$ to more recent investigations demonstrating a true continuous change in density, ${ }^{19,25-27}$ superior energy absorption capabilities were noticed compared with their uniform counterparts. This is partially associated with a sequential failure of consecutive lattice layers.

Considering multiphysical problems-towards which lattices are generally geared-FGLs have potential to locally tailor, e.g., the permeability or energy absorption while also fulfilling some structural functions. Understanding and determining the effect of grading on the performance of a specific unit cell with a certain relative density is therefore vital for applications of and designs with lattices.

\section{Fiber-Reinforced Additive Manufacturing (FRAM)}

As AM has moved progressively from prototyping to fabrication, FRAM and the use of high-performance materials with short and continuous fiber reinforcements ${ }^{28-37}$ have drawn increasing attention in the pursuit of increasing the specific stiffness of AM parts. The consideration of infill patterns and the placement of fibers has thereby become more important, as the in-plane anisotropy is exacerbated. In the context of latticing, FRAM can potentially mitigate the loss in stiffness compared with solid counterparts and is therefore of interest for this study.

\section{Scope of This Paper}

The work presented herein sheds light on the effect of grading on the energy absorption behavior and performance of strut- and surface-based lattices. Fused deposition modeling (FDM) with short carbonfiber-reinforced polymer is applied to fabricate the well-known strut-based BCC and surface-based Schwarz-P lattices with three dissimilar severities of grading but the same average volume fraction (mean density between maximum and minimum). Uniaxial compression tests were conducted with homogeneous (ungraded) pendants serving as the baseline. The deformation mechanisms were also recorded to provide further insight into the energy absorption curves. The power-law approach is employed to retrieve material parameters to predict the performance as a function of grading.

\section{METHODOLOGY}

\section{Modeling Functionally Graded (FG) Lattices}

FG-TPMS lattices were generated implicitly following the work of Panesar et al. ${ }^{19}$ This work

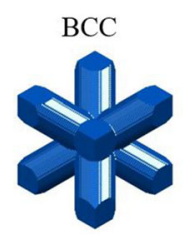

Schwarz-P

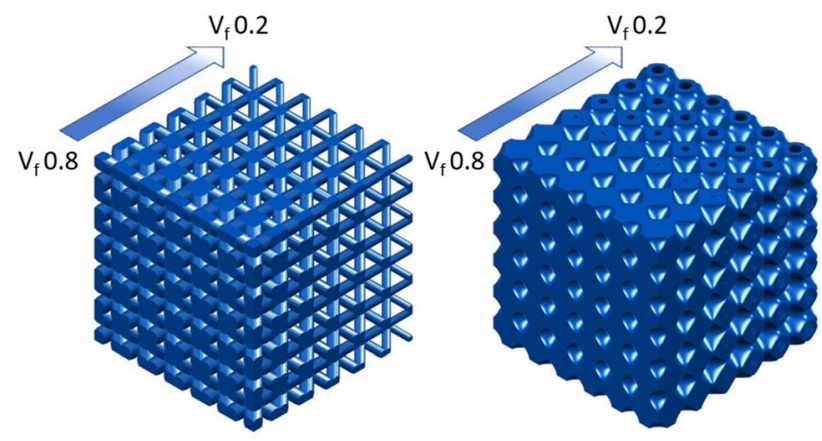

Fig. 1. Continuously and linearly graded BCC and Schwarz-P lattices with different severities in grading between two opposing surfaces of cubic test specimens.

focuses on the strut-based BCC lattice and surfacebased Schwarz-P lattice, represented in a sheet or "double" variant. Previous studies have shown convergence of the elastic modulus with an increased number of unit cells in the test configuration. ${ }^{38}$ Based on these findings and earlier experimental work on FG-TPMS by Maskery et al., ${ }^{23}$ cubic test specimens with dimensions of $30 \mathrm{~mm} \times$ $30 \mathrm{~mm} \times 30 \mathrm{~mm}$ composed of $6 \times 6 \times 6$ unit cells were investigated.

Following the work of Panesar et al.,${ }^{19}$ the surface representation with iso-value offset $t$, defining the extent of dilation of the midplane shell representation, was used. The zero-surface (outside versus inside) function for the Schwarz-P (SP) TPMS lattice is defined by the equation

$$
f_{p}(x, y, z)=\cos \left(\lambda_{x} t\right)+\cos \left(\lambda_{y} t\right)+\cos \left(\lambda_{z} t\right),
$$

where $\lambda_{i}$ stands for the three-dimensional (3D) periodicity and is calculated as $2 \pi \times n_{i} / L_{i}\left(n_{i}\right.$ representing the number of tessellations and $L_{i}$ the lattice dimension).

A linear grading scheme between the two opposing surfaces of the coupon was chosen for this work (Fig. 1). The grading is hereby realized through a four-dimensional (4D) representation $(x, z, y, t)$, as described in Ref. 19, whereby the iso-value regulates the volume fraction $V_{\mathrm{f}}$. Three different severities of grading were tested, while the average volume fraction (mean density between extremes) was kept constant at $V_{\mathrm{f}}=0.5$ : (1) $0.8-0.2$, (2) $0.7-$ 0.3 , (3) $0.6-0.4$, and (4) ungraded 0.5 as reference.

\section{Material and Fabrication}

The MarkTwo 3D printer from Markforged Inc. was employed in conjunction with the slicing software Eiger (version 2.2.0) to print the test coupons. 


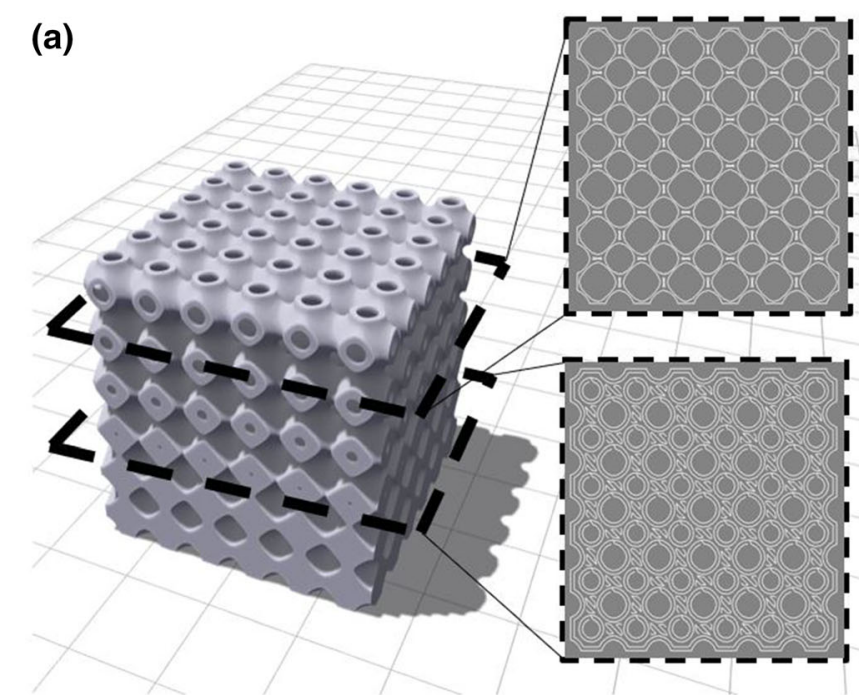

(b)

Fig. 2. (a) Build direction regarding the direction of grading with screenshots of slices at different heights revealing the chosen build parameters and (b) printed SFR specimens.
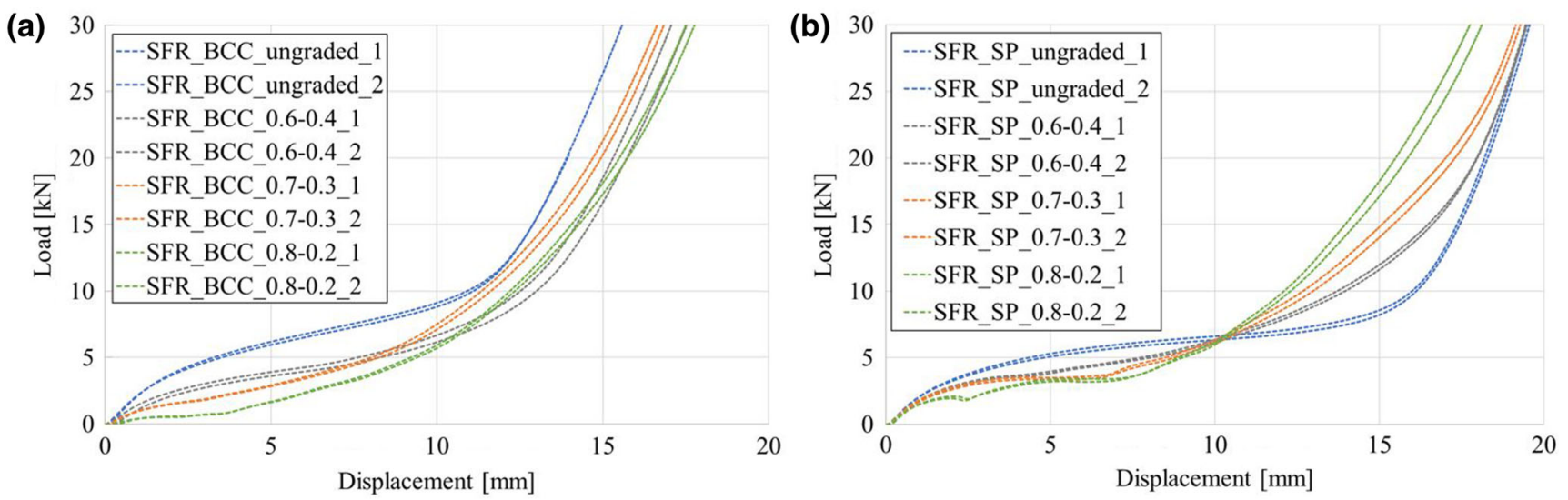

Fig. 3. Load-displacement curves for (a) BCC and (b) Schwarz-P (SP) lattices tested with various severities of grading (Color figure online).

Parts were printed using short carbon-fiber-reinforced (SFR) nylon (Onyx ${ }^{39}$ ) with the build direction in line with decreasing unit cell volume fraction (Fig. 2). Two layers were chosen for the walls (i.e., shell) as well as the roof and floor, while supports were deselected to avoid difficulties in removal. All specimens were printed with a solid infill, i.e., $\pm 45^{\circ}$ deposition path pattern, for consecutive layers, and the average specimen weight was $13.55 \pm 0.16 \mathrm{~g}$. Note that the average weight difference between the BCC and SP specimen was found to be $2.4 \%$ after printing.

\section{Mechanical Testing}

Uniaxial compression tests were conducted using an Instron 5969 universal testing machine equipped with a $50-\mathrm{kN}$ load cell. Tests were carried out at strain rate of $0.5 \mathrm{~mm} / \mathrm{min}$, following ASTM C365/ C356M-16 standard. ${ }^{40}$ A speckle pattern was applied on the front surface of the specimens to monitor the average unit cell layer strain parallel to the loading direction using an optical strain gauge (Imetrum Ltd.). Two specimens were tested for each cell type and density gradient. The nomenclature used for the test specimens is as follows:

- [Reinforcement type]_[Unit cell type]_[Gradient from max-min]_[Specimen number] (e.g., SFR_BCC_0.7-0.3_1)

\section{RESULTS AND DISCUSSION}

\section{Experimental Results}

Figure 3 illustrates the elastoplastic load-displacement curves for the BCC and SP lattice under uniaxial compression. In the BCC batch, a plastic plateau was only noticed for the most severely graded specimens, whereas the SP lattices displayed plateaus except for the specimen with grading of $0.6-0.4$. A distinct load drop-off between $7 \%$ and $10 \%$ lattice strain $(\sim 2-3 \mathrm{~mm}$ displacement $)$ 

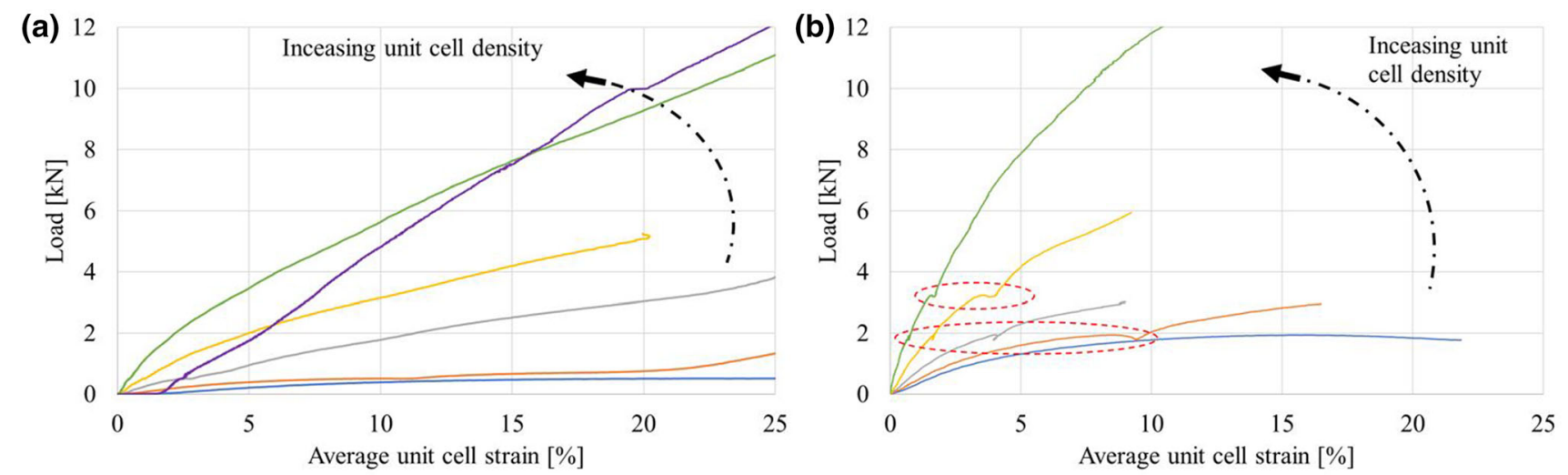

Fig. 4. Average unit cell strain for (a) BCC and (b) SP lattice with most severe density grading recorded by an optical strain gauge. Failure events are highlighted with red circles (Color figure online).

was experienced in the SP lattices with grading from 0.8 to 0.2 , indicating a clear instability such as, e.g., an isolated layer collapse. This is followed by a pronounced load plateau between $16 \%$ and $22 \%$ lattice strain $(\sim 5-7 \mathrm{~mm}$ displacement). The BCC lattice with the same grading, on the contrary, exhibited no load drop but only a plateauing load between $6 \%$ and $8 \%$ lattice strain $(\sim 1.8-2.4 \mathrm{~mm}$ displacement), suggesting typical bending-dominated deformation.

The SP lattices exhibited a point of intersection at displacement of about $11 \mathrm{~mm}$, after which the specimens with higher grading experience higher loading for the same displacement, whereas the BCC lattices with the lowest grading (i.e., ungraded) constantly showed higher loads for the same strain, as for the SP lattice before the intersection strain. Compared with the work of, e.g., Maskery et al., ${ }^{23}$ in which unreinforced nylon specimens with stepwise linear grading (each unit cell layer having a set mean relative density) exhibited distinct sequential failure, this work investigated a reinforced structure with true linear grading, demonstrating a more gradual failure. The load-displacement curve shown in Fig. 3 highlights this, as even the more severely graded structures display a smooth trend.

As the most severely graded BCC and SP lattice displayed a more distinct response in the loaddisplacement curve, their average unit cell strain was processed (Fig. 4) to elucidate the differences in the deformation mechanisms and the degree of load bearing in each layer throughout the loading process. For the BCC and SP lattice, six and five equidistant strain measurements equal to the edge length of a unit cell were taken. For the same load and comparable unit cell density, BCC cells were strained almost twice as much as the SP lattices, indicating a lower effective stiffness as expected from bending-dominated versus stretching-dominated unit cell types. This can be seen, e.g., when comparing the highest-density unit cell layers in Fig. 4, highlighted in green. Additionally, the surface-based lattices exhibited load drops in the realm of $2 \mathrm{kN}$ and $3 \mathrm{kN}$ throughout unit cell layers of different density, if the layer had not yet failed or started to yield (see, e.g., the lowest unit cell density measurement in Fig. 4b). This implies stretchingdominated behavior due to the presence of local instabilities caused, e.g., by buckling and that the force was more evenly distributed in the SP corresponding to the global structural response to a sudden local event. In this context, it is also noteworthy that layers $1-3$ and 4 and 5 of the SP lattice deformed in a similar fashion (Fig. 7). In contrast, the BCC lattice was more bending dominated and did not display local instability. Furthermore, note that the BCC lattice yielded a lower stiffness compared with the SP lattice.

\section{Effect of Grading on Energy Absorption and Deformation Mechanisms}

Figure 5 displays the cumulative energy absorption per unit area during the uniaxial compression test (as derived from the load-displacement curve). Compared with the BCC lattices, the SP batch displayed a much narrower distribution of the trendlines, with a noticeable point of intersection at about $48 \%$ lattice strain. This means that the grading in the BCC lattices resulted in a much greater reduction in energy absorption, whereas this was less pronounced for the surface-based counterpart. In fact, the energy absorption of the SP lattices became greater with more severe grading after exceeding the intersection strain. It can be observed that the energy absorption of the ungraded BCC lattice was always greater than that of the SP lattice after about 20\% lattice strain (at lower strains, the values were similar). Vice versa, for the most severe grading, the SP lattices performed better, independent of the lattice strain. On the contrary, the energy absorption behavior was very similar for the two lattice types with grading of 0.60.4 and $0.7-0.3$ up to about $45 \%$ lattice strain. Only at higher strains did the strut-based lattice outperform its surface-based counterpart. 
(a)

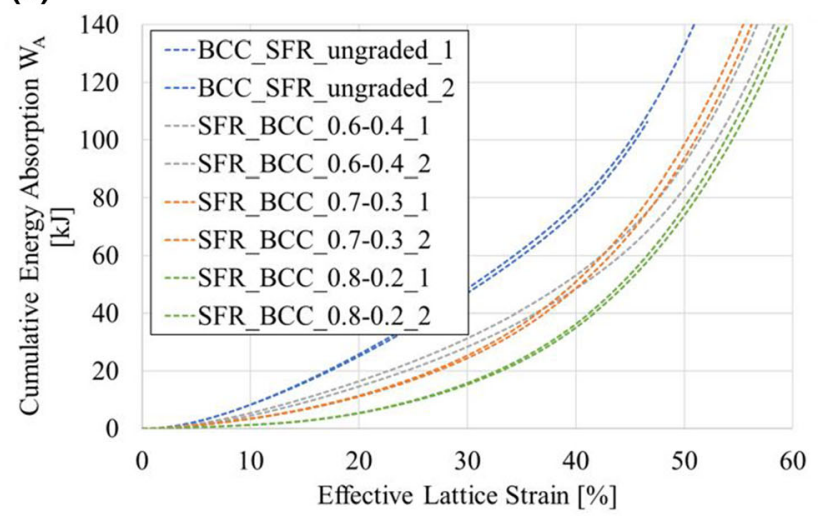

(b)

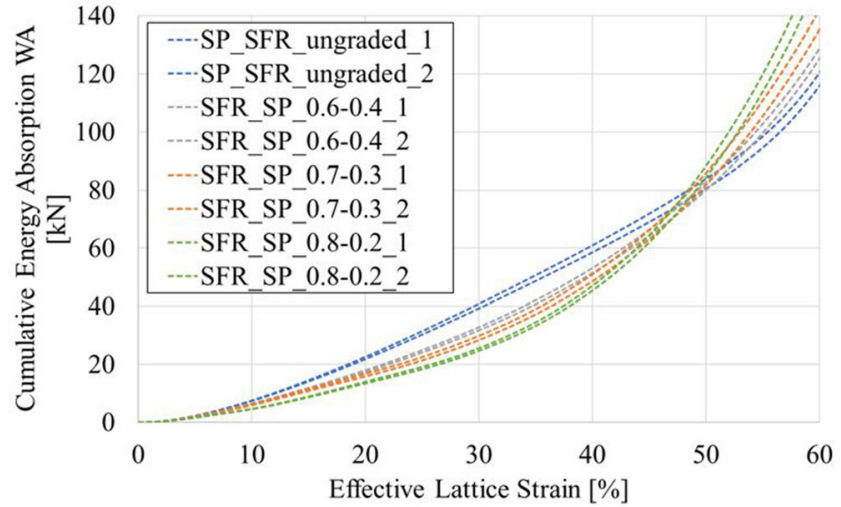

Fig. 5. Cumulative energy absorption curve for (a) BCC and (b) SP lattices tested with various severities of grading (Color figure online).
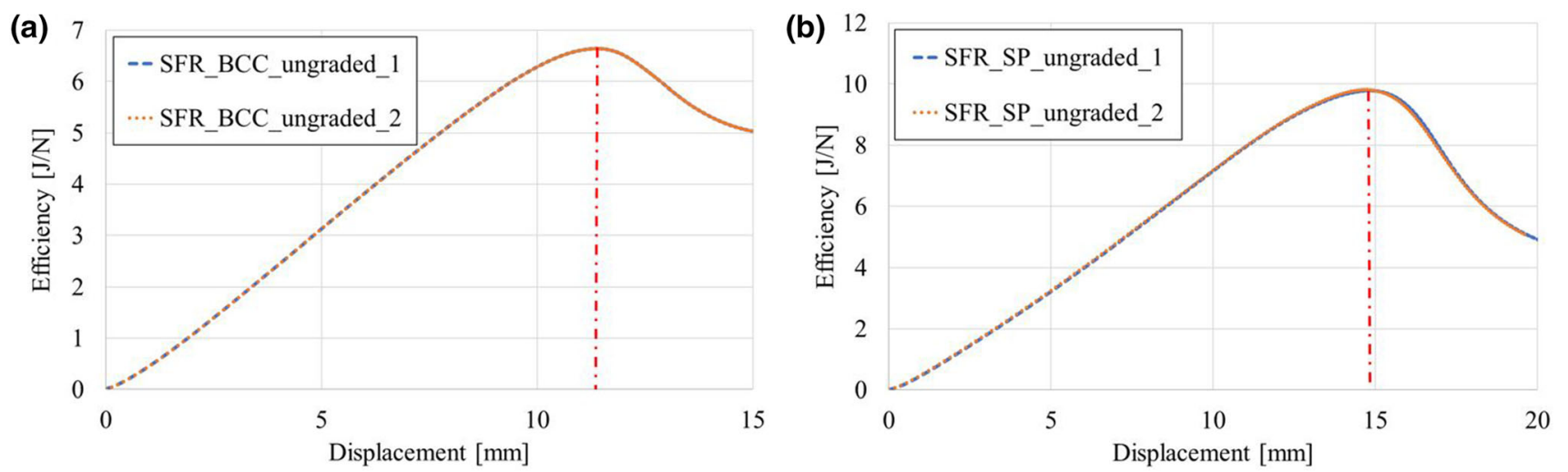

Fig. 6. Efficiency-displacement curve for uniform (a) BCC and (b) SP lattices (Color figure online).

(a)

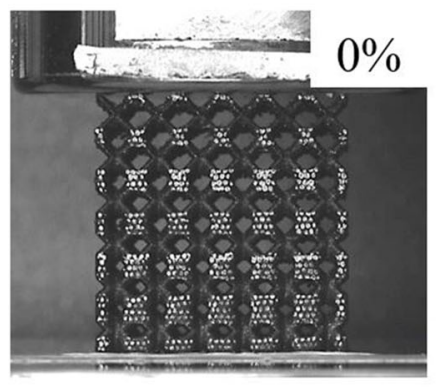

(b)

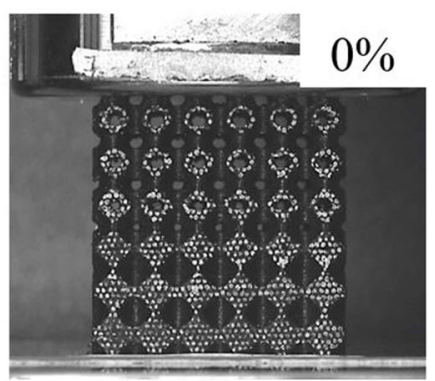

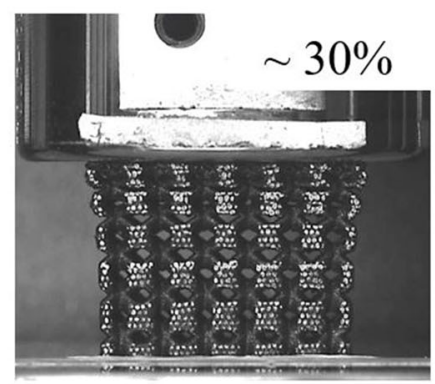
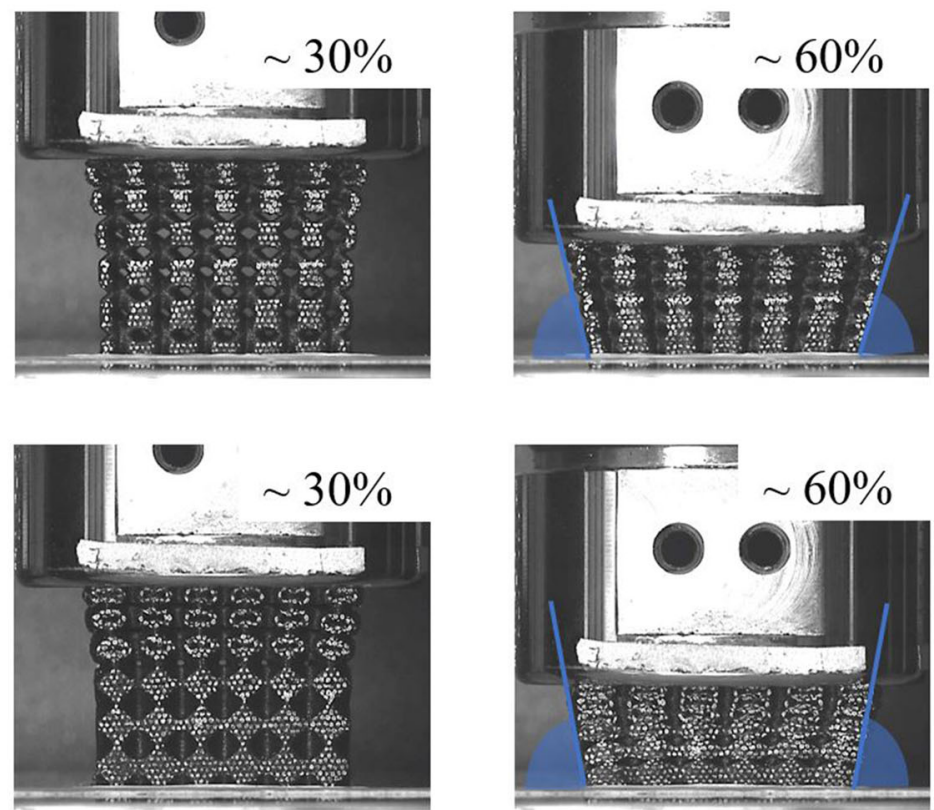

Fig. 7. Screenshots of uniaxial compression test of (a) BCC and (b) Schwarz-P lattices with grading from 0.8 to 0.2. The transformation of the test cube into a trapezoidal shape becomes more distinct with higher strain, as highlighted in blue (Color figure online). 


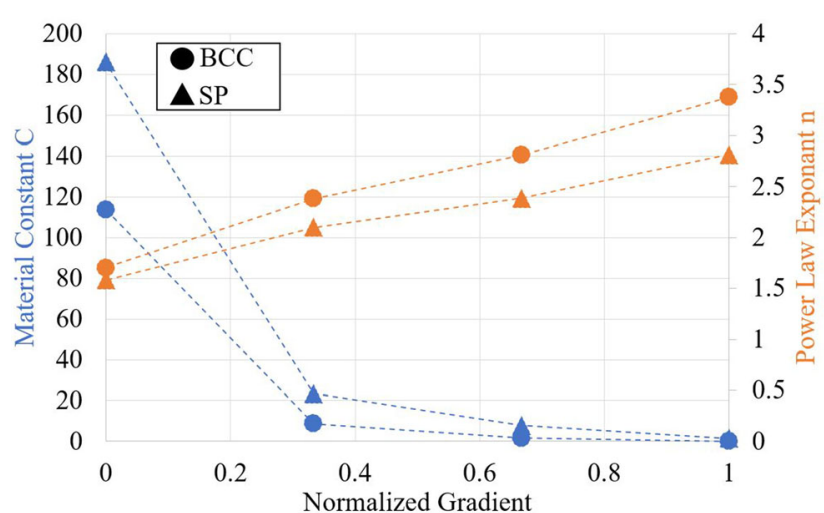

Fig. 8. Power-law constant and exponent for energy absorption of lattices with different severity of grading but constant relative density (Color figure online).

For the ungraded, i.e., uniform lattices, the energy efficiency method, as introduced by $\mathrm{Li}$ et al., ${ }^{41}$ was used to determine the densification onset strain, which was found to produce consistent results for the point at which cell walls begin to interact, leading to an increased compressive load (Fig. 6, dotted red line). The BCC lattice started to densify much earlier at around $38 \%$ strain with lower maximum efficiency, compared with the SP lattice, which reached the highest efficiency at about $50 \%$. This means that the SP lattice absorbed $\sim 23 \%$ more energy up to the densification strain than the BCC pendant. For the graded specimens, this energy efficiency method was found to be unsuitable, requiring further investigations into how to define this point.

The deformation stages for the specimens with the highest degree of grading are illustrated in Fig. 7. The deformation zones of the SP and BCC specimens with a grading of $0.8-0.2$ were clearly split between the first and last three cell layers, whereas only above $30 \%$ strain did the lower unit cell rows start to deform visually. The specimens adopted a trapezoidal shape during deformation, as the low-density unit cells started to strain more severely perpendicular to the loading direction. In contrast, a barrel-like shape was found for the ungraded specimens, indicating higher lateral stresses in the mid-region.

Similar to previous studies, ${ }^{20,25,26}$ a power law was employed to fit the curves for the energy absorption behavior of the functionally graded lattices. The absorbed energy $W_{\mathrm{A}}^{\text {eff }}$ was determined as follows:

$$
W_{\mathrm{A}}^{\mathrm{eff}}=C \times\left(\varepsilon_{\text {lattice }}^{\text {eff }}\right)^{n}
$$

with $C$ being a material constant, $\varepsilon_{\text {lattice }}^{\text {eff }}$ the effective lattice strain, and $n$ the exponent. Figure 8 illustrates the results for the $C$ and $n$ values as functions of the normalized gradient and the lattice type for constant relative density. While more data points are needed to fit curves onto the individual constants, a similar trend for the BCC and SP lattices becomes apparent. Further investigations shall shed light on these trendlines to provide a reliable database from which material properties could be easily derived.

\section{CONCLUSION}

The energy absorption capabilities of short fiberreinforced lattices are outlined as a function of grading severity and unit cell type. Both surfaceand strut-based (BCC and Schwarz-P) lattices with continuous grading were fabricated using FDM and tested in uniaxial compression. Ungraded and slightly graded (linear density variation from 0.6 to 0.4 and from 0.7 to 0.3 ) BCC lattices were found to absorb more energy for given strain than their SP counterparts, except for the most severely graded SP lattice (with a linear density variation from 0.8 to 0.2 ), which outperformed the strut-based specimen. Dissimilar deformation behaviors were found between the two types of unit cell with severe grading. The BCC lattices demonstrated a bendingdominated response with steady yielding of all layers, resulting in a lower stiffness compared with the SP lattice, which exhibited load drops across all layers in highly graded specimens, indicating local instabilities caused by buckling. A power-law approach was used to capture the materials' energy absorption capability for fixed relative density and unit cell type as a function of the gradient. The findings of this work should provide engineers and designers valuable insight into the properties of functionally graded lattices, paving the way for increased uptake in the design of additive manufacturing procedures.

Future works could investigate how these reinforced specimens compare with their unreinforced pendants, and what effect the infill pattern has on the final properties. As methods such as the energy efficiency approach no longer hold for the determination of the densification onset of graded lattices, studies into equivalent and universal indicators for the response of the structure would also be worthwhile.

\section{OPEN ACCESS}

This article is licensed under a Creative Commons Attribution 4.0 International License, which permits use, sharing, adaptation, distribution and reproduction in any medium or format, as long as you give appropriate credit to the original author(s) and the source, provide a link to the Creative Commons licence, and indicate if changes were made. The images or other third party material in this article are included in the article's Creative Commons licence, unless indicated otherwise in a credit line to the material. If material is not included in the article's Creative Commons licence and your intended use is not permitted by statutory 
regulation or exceeds the permitted use, you will need to obtain permission directly from the copyright holder. To view a copy of this licence, visit http://creativecommons.org/licenses/by/4.0/.

\section{REFERENCES}

1. L. Chougrani, J.P. Pernot, P. Véron, and S. Abed, $C A D$ Comput. Aided Des. 90, 95 (2017).

2. M. Eichenhofer, J.C.H. Wong, and P. Ermanni, Addit. Manuf. 18, 48 (2017).

3. P. Terriault and V. Brailovski, Finite Elem. Anal. Des. 138, 1 (2018).

4. A. Clausen, N. Aage, and O. Sigmund, Engineering 2, 250 (2016).

5. M. Helou and S. Kara, Int. J. Comput. Integr. Manuf. 31, 243 (2017).

6. E. Handler, A. Sterling, J. Pegues, H. Ozdes, and M. Masoomi, in Proceedings 28th Annual International Freeform Fabrication Symposium (Austin, Texas, 2017), p. 2632.

7. A.O. Aremu, J. Brennan-Craddock, A. Panesar, I.A. Ashcroft, R.J.M. Hague, R.D. Wildman, and C. Tuck, Addit. Manuf. 13, 1 (2017).

8. T. Tancogne-Dejean, A.B. Spierings, and D. Mohr, Acta Mater. 116, 14 (2016)

9. P. Qiao, M. Yang, and F. Bobaru, J. Aerosp. Eng. 21, 235 (2008).

10. M. McMillan, M. Jurg, M. Leary, and M. Brandt, Procedia Technol. 20, 178 (2015).

11. MSRI (Mathematical Sciences Research Institute), Triply Periodic Minimal Surfaces-Scientific Graphic Project, htt p://www.msri.org/publications/sgp/jim/papers/morphbysym metry/table/index.html. Accessed 13 June 2019.

12. Materialise, Materialise 3-matic Lightweight Structures Module, http://www.materialise.com/en/software/3-matic/m odules/lightweight-structures-module, Accessed 14 Dec 2017.

13. Altair, Altair OptiStruct ${ }^{\circledR}$ Revolutionizes Lattice Structures for $3 D$ Printing, http://www.altairhyperworks.com/newsdet ail.aspx?news_id=11109\&news_country=en-US, Accessed 14 Dec 2017.

14. nTopology, Architected Materials, https://ntopology.com/arc hitected-materials/, Accessed 19 Nov 2019.

15. Betatype Ltd., Betatype-We Deliver on the Promise of Thin Wall Surface Structures Through Additive Manufacturing, http://www.betaty.pe/case-studies/surface-structures/, Accessed 26 Jan 2019

16. L. Han and S. Che, Adv. Mater. 30, 1 (2018).

17. I. Maskery, N.T. Aboulkhair, A.O. Aremu, C.J. Tuck, and I.A. Ashcroft, Addit. Manuf. 16, 24 (2017).

18. S. Rajagopalan and R.A. Robb, Med. Image Anal. 10, 693 (2006).
19. A. Panesar, M. Abdi, D. Hickman, and I. Ashcroft, Addit. Manuf. 19, 81 (2018).

20. L. Zhang, S. Feih, S. Daynes, S. Chang, M.Y. Wang, J. Wei, and W.F. Lu, Addit. Manuf. 23, 505 (2018).

21. I. Maskery, A.O. Aremu, L. Parry, R.D. Wildman, C.J. Tuck, and I.A. Ashcroft, Mater. Des. 155, 220 (2018).

22. R. Gabbrielli, I.G. Turner, and C.R. Bowen, Key Eng. Mater. 361-363, 903 (2009)

23. I. Maskery, A. Hussey, A. Panesar, A. Aremu, C. Tuck, I. Ashcroft, and R. Hague, J. Cell. Plast. 53, 151 (2017).

24. M. Afshar, A.P. Anaraki, H. Montazerian, and J. Kadkhodapour, J. Mech. Behav. Biomed. Mater. 62, 481 (2016)

25. S.Y. Choy, C.N. Sun, K.F. Leong, and J. Wei, Mater. Des. 131, 112 (2017).

26. D.S.J. Al-Saedi, S.H. Masood, M. Faizan-Ur-Rab, A. Alomarah, and P. Ponnusamy, Mater. Des. 144, 32 (2018).

27. L. Yang, R. Mertens, M. Ferrucci, C. Yan, Y. Shi, and S. Yang, Mater. Des. 162, 394 (2019).

28. S. Liu, Y. Li, and N. Li, Mater. Des. 137, 235 (2018).

29. P. Zhuo, S. Li, I. Ashcroft, A. Jones, and J. Pu, in 21st International Conference on Composite Materials (2017).

30. L. G. Blok, B. K. S. Woods, H. Yu, M. L. Longana, and K. P. Potter, in 21st International Conference on Composite Materials (2017).

31. A.N. Dickson, J.N. Barry, K.A. McDonnell, and D.P. Dowling, Addit. Manuf. 16, 146 (2017).

32. B.G. Compton and J.A. Lewis, Adv. Mater. 26, 5930 (2014).

33. M. Ivey, G.W. Melenka, J.P. Carey, and C. Ayranci, Adv. Manuf. Polym. Compos. Sci. 3, 81 (2017).

34. J.J. Martin, B.E. Fiore, and R.M. Erb, Nat. Commun. 6, 1 (2015).

35. L.R. Holmes and J.C. Riddick, JOM 66, 270 (2014).

36. S. Christ, M. Schnabel, E. Vorndran, J. Groll, and U. Gbureck, Mater. Lett. 139, 165 (2015).

37. Z. Quan, A. Wu, M. Keefe, X. Qin, J. Yu, J. Suhr, J.H. Byun, B.S. Kim, and T.W. Chou, Mater. Today 18, 503 (2015).

38. I. Maskery, L. Sturm, A.O. Aremu, A. Panesar, C.B. Williams, C.J. Tuck, R.D. Wildman, I.A. Ashcroft, and R.J.M. Hague, Polymer (Guildf) 152, 62 (2018).

39. Markforged, Inc., Composite 3D Printing, https://markforg ed.com/composites/. Accessed 14 Dec 2017.

40. ASTM C365/C365M-16, Standard Test Method for Flatwise Compressive Properties of Sandwich Cores (West Conshohocken, PA: ASTM International, 2016). https://doi. org/10.1520/C0365_C0365M-16.

41. Q.M. Li, I. Magkiriadis, and J.J. Harrigan, J. Cell. Plast. 42, 371 (2006).

Publisher's Note Springer Nature remains neutral with regard to jurisdictional claims in published maps and institutional affiliations. 